\title{
NONLINEAR SURFACE WAVES ON A TANGENTIAL DISCONTINUITY IN MAGNETOHYDRODYNAMICS
}

\author{
BY \\ GIUSEPPE ALÌ (Institute for Applied Mathematics, Consiglio Nazionale delle Ricerche, Napoli) \\ AND
}

JOHN K. HUNTER (Department of Mathematics and Institute of Theoretical Dynamics, University of California at Davis)

\begin{abstract}
We derive an asymptotic equation that describes the propagation of weakly nonlinear surface waves on a tangential discontinuity in incompressible magnetohydrodynamics. The equation is similar to, but simpler than, previously derived asymptotic equations for weakly nonlinear Rayleigh waves in elasticity, and is identical to a model equation for nonlinear Rayleigh waves proposed by Hamilton et al. The most interesting feature of the surface waves is that their nonlinear self-interaction is nonlocal. As a result of this nonlocal nonlinearity, smooth solutions break down in finite time, and appear to form cusps.
\end{abstract}

1. Introduction. Surface wave solutions of hyperbolic systems of partial differential equations on a half-space are waves that propagate along the boundary of the half-space and decay exponentially into the interior. Rayleigh waves in elasticity are an important physical example. Surface waves may also propagate on discontinuities, such as contact discontinuities or shock waves, in solutions of systems of conservation laws. The purpose of this paper is to study a prototype problem for nonlinear surface wave propagation on a discontinuity, namely the propagation of surface waves on a tangential discontinuity in magnetohydrodynamics (MHD).

In addition to the specific aim of studying MHD surface waves, a general aim of this work is the analysis of nonlinear boundary value and discontinuity problems for conservation laws that satisfy a Lopatinski condition, which is necessary for local wellposedness, but not a uniform Lopatinski condition, which is sufficient for local wellposedness. As we explain in Sec. 2, the failure of the uniform Lopatinski condition is typically caused by the presence of surface waves.

Received January 24, 2001.

2000 Mathematics Subject Classification. Primary 35L60, 74J30, 76W05.

Key words and phrases. Nonlinear surface waves, magnetohydrodynamics.

The second author was partially supported by the NSF under grant number DMS-0072343.

(C)2003 Brown University 
We consider incompressible MHD for simplicity. The corresponding conservation laws are not hyperbolic, but a similar analysis would apply to the hyperbolic compressible MHD equations, and to other systems of conservation laws that have surface wave solutions. For example, Benzoni-Gavage [4] observed that exponentially decaying surface waves can appear on propagating phase boundaries in a van der Waals fluid.

A tangential discontinuity in MHD is a surface across which there are jumps in the tangential components of the fluid velocity and the magnetic field, and the normal component of the magnetic field vanishes. We denote appropriately nondimensionalized fluid velocities and magnetic fields on either side of the tangential discontinuity by $\mathbf{v}^{ \pm}$and $\mathbf{B}^{ \pm}$, respectively. A planar tangential discontinuity is linearly stable [13] if

$$
\frac{1}{2}\left|\mathbf{v}^{+}-\mathbf{v}^{-}\right|^{2}<\left|\mathbf{B}^{+}\right|^{2}+\left|\mathbf{B}^{-}\right|^{2} \text {. }
$$

For example, if the velocity is continuous, then the tangential discontinuity is stable; and if the velocity is discontinuous, then the tangential discontinuity is subject to a Kelvin-Helmholtz instability when the magnetic field is sufficiently small compared with the jump in the tangential velocity, but a sufficiently large magnetic field stabilizes the discontinuity.

A surface wave can propagate along the tangential discontinuity when it is linearly stable. We measure the amplitude of the surface wave by the normalized displacement $\varphi(x, t)$ of the discontinuity in a reference frame moving with the linearized phase velocity of the wave. Our main result is that the displacement of a weakly nonlinear surface wave satisfies a "nonlocal Hamilton-Jacobi equation"

$$
\varphi_{t}+\frac{1}{2}\left(\mathbb{H}\left[(\mathbb{H}[\varphi])^{2}\right]\right)_{x x}+\mathbb{H}[\varphi] \varphi_{x x}=0
$$

where $\mathbb{H}$ denotes the Hilbert transform. As we discuss below, Hamilton et al. [6] proposed this equation as a model equation for nonlinear Rayleigh waves, and it is interesting that exactly their model equation arises for surface waves on a tangential discontinuity in incompressible MHD.

Equation (1.2) has a different structure from well-known nonlocal evolution equations for nonlinear dispersive waves, such as the Benjamin-Ono equation, whose potential form is

$$
\varphi_{t}+\frac{1}{2} \varphi_{x}^{2}+\mathbb{H}[\varphi]_{x x}=0
$$

Equation (1.2) is nondispersive and the nonlinear term is a nonlocal function of the solution $\varphi$, whereas (1.3) is dispersive and the linear dispersive term is a nonlocal function of $\varphi$.

We may write $(1.2)$ in the spectral form $[1,6]$

$$
\psi_{t}(k, t)+i \operatorname{sgn} k \int_{-\infty}^{+\infty} \Lambda(k-l, l) \psi(k-l, t) \psi(l, t) d l=0,
$$

where $\psi(k, t)$ is the Fourier transform of the displacement $\varphi(x, t)$, and the kernel $\Lambda$ is given by

$$
\Lambda(k, l)=\frac{2|k+l||k||l|}{|k+l|+|k|+|l|} .
$$

In fact, the asymptotic expansion leads directly to the spectral form of the equation. 
Previous studies of nonlinear surface waves on discontinuities include the work of Artola and Majda [2, 3] for compressible vortex sheets, and the work of Majda and Rosales $[19,20]$ for detonation waves. The surface waves in these problems radiate bulk waves into the interior of the fluid, and thus they differ qualitatively from genuine surface waves, such as Rayleigh waves, whose energy is localized at the surface and decays exponentially away from it. We discuss the distinction between these radiative surface waves and genuine surface waves further in the next section. As far as we know, this work is the first study of nonlinear, exponentially decaying surface waves on a discontinuity.

In the compressible vortex-sheet problem, Artola and Majda [2] showed that the displacement $\varphi(x, t)$ of the vortex sheet satisfies a local Hamilton-Jacobi equation,

$$
\varphi_{t}+\frac{1}{2} \varphi_{x}^{2}=0
$$

In this problem, there are three different surface wave modes. Artola and Majda [3] also derived a nonlocal equation that describes resonant interactions between these modes, and showed that it leads to a nonlinear instability in the vortex sheet. The origin of this nonlocality is the resonant interaction of the bulk waves generated by different surface waves in the interior of the fluid. Here we consider the propagation of a single weakly nonlinear surface wave, and we do not analyze the interaction between different surface waves.

In the detonation wave problem, Majda and Rosales $[19,20]$ obtained a nonlocal equation for the perturbation $\varphi(x, t)$ in the location of the detonation wave associated with a single surface wave,

$$
\varphi_{t}+\frac{1}{2} \varphi_{x}^{2}+\alpha \int_{0}^{\infty} \varphi_{x}(x+\beta s) \varphi_{x x}(x+s) d s=0
$$

This equation has some similarities with (1.2), but its detailed structure and the qualitative properties of its solutions, such as the nature of their singularities, are not the same. Moreover, the physical origin of the nonlocality is different: the surface wave radiates bulk waves into the interior of the fluid, and the resonant interaction of these waves in the interior of the fluid generates another bulk wave, which propagates back to the detonation wave and disturbs it [24].

Asymptotic equations describing the propagation of weakly nonlinear Rayleigh waves on a half-space were derived in $[5,6,7,10,11,14,15,22,23,29]$, and the references cited there. Similar equations, in both spectral and spatial forms, for surface waves on a half-space governed by general first order hyperbolic systems were derived in [9]. The asymptotic equation for weakly nonlinear Rayleigh waves on a half-space occupied by an isotropic, hyperelastic solid may be written in the spectral form (1.4) with the kernel 
(see $[5,22]$, for example)

$$
\begin{aligned}
\Lambda(k, l)= & \frac{\alpha|k+l||k||l|}{|k+l|+r|k|+r|l|}+\frac{\alpha|k+l||k||l|}{r|k+l|+|k|+r|l|} \\
& +\frac{\alpha|k+l||k||l|}{r|k+l|+r|k|+|l|}+\frac{\beta|k+l||k||l|}{r|k+l|+|k|+|l|} \\
& +\frac{\beta|k+l||k||l|}{|k+l|+r|k|+|l|}+\frac{\beta|k+l||k||l|}{|k+l|+|k|+r|l|} \\
& +\frac{\gamma|k+l||k||l|}{|k+l|+|k|+|l|} .
\end{aligned}
$$

Here, $r=c_{t} / c_{l}$ is the ratio of the solid's transverse and longitudinal wave speeds, $c_{t}$ and $c_{l}$ respectively, and $\alpha, \beta, \gamma$ are real constants. The kernel (1.8) reduces to (1.5) when $\alpha=\beta=0$, which is what led Hamilton et. al. [6] to propose (1.2) as a model equation for nonlinear Rayleigh waves.

If the governing equation of a nonlinear wave is Hamiltonian, then we expect that an asymptotic equation for the wave is also Hamiltonian, provided that we expand with respect to canonical variables [21]. The Hamiltonian structure of the asymptotic equation for nonlinear Rayleigh waves was observed in [7], and the Hamiltonian structure of the asymptotic equations for general Hamiltonian surface waves is discussed in [1]. The fact that the asymptotic equation is Hamiltonian provides a useful check on the lengthy algebra required to derive it from the system of MHD equations. It should be possible to derive the same equation by expansion of an appropriate surface-wave Hamiltonian, but we do not follow that approach here.

A common theme in nonlinear wave propagation is the formation of finite-time singularities. Smooth solutions of the local Hamilton-Jacobi equation (1.6) typically break down in finite time. The resulting singularities correspond to the formation of corners, or "kinks," in the free surface, as in the case of compressible vortex sheets $[2,3]$. Solutions of (1.7) also form corners, something that is interpreted in $[19,20]$ as the formation of Mach stems on the detonation wave.

The nonlinear distortion of Rayleigh waves has been studied extensively (see [6], [11], [22], and the references cited there). Smooth solutions of (1.2), as well as of the corresponding equation for nonlinear Rayleigh waves, also appear to break down in finite time. In Fig. 1, we show a numerical solution of (1.2) with cosine initial data,

$$
\varphi(x, 0)=\cos 2 \pi x,
$$

computed using a spectral viscosity method [28]. The solution is similar to, but more resolved than, the one shown in [6]. We also show in Fig. 1 a plot of

$$
\mathcal{P}=-\frac{1}{4 \pi} \int_{0}^{1} \mathbb{H}[\varphi] \varphi_{x} d x
$$

as a function of time. This quantity is conserved for smooth solutions of (1.2). The conservation of $\mathcal{P}$ is associated with the invariance of (1.2) under spatial translations, so we may interpret $\mathcal{P}$ as the momentum of the wave. A singularity forms at $t \approx 0.5$, after which the momentum decreases. The solution appears to remain continuous, but a cusp 
forms where its derivative blows up, unlike solutions of (1.6) and (1.7) whose derivatives remain essentially bounded.

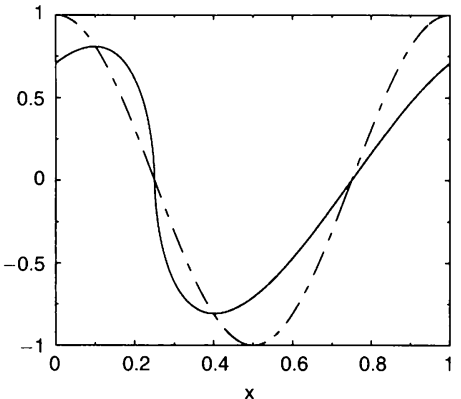

(a)

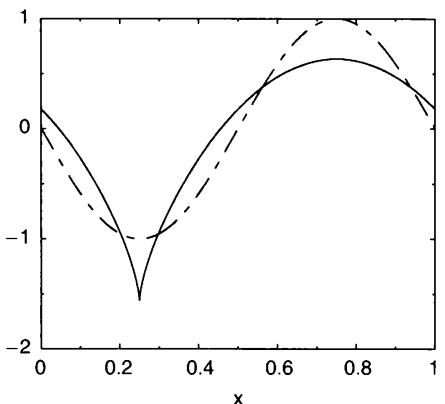

(c)

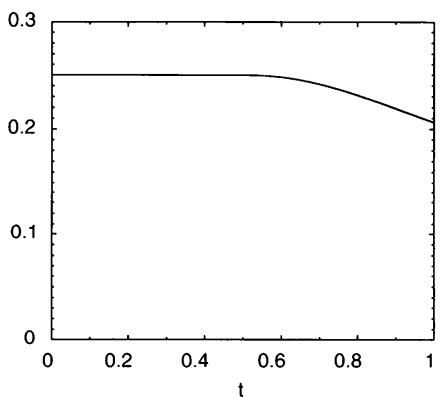

(e)

FIG. 1. A spectral-viscosity solution of (1.2) with the harmonic initial data (1.9): (a) the solution $\varphi$ at time $t=1$ (the initial data is dashed); (b) the derivative $\varphi_{x}$ at time $t=1$; (c) the Hilbert transform $\mathbb{H}[\varphi]$ of the solution at time $t=1$ (the initial data is dashed); (d) the derivative $\mathbb{H}\left[\varphi_{x}\right]$ of the Hilbert transform at $t=1$; (e) the momentum $\mathcal{P}$ defined in (1.10) as a function of time; (f) the spectrum $\log |\psi(k, t)|$ at time $t=1$, where $\psi$ gives the Fourier coefficients of $\varphi$. We use the Fourier modes $e^{2 \pi i k x}$ with $|k| \leq N$, where $\mathrm{N}=$ 4096. In the spectral viscosity method, we use a small viscous dissipation with coefficient of viscosity $1 / N$, that acts on the Fourier modes with $|k| \geq 2 \sqrt{N}$.

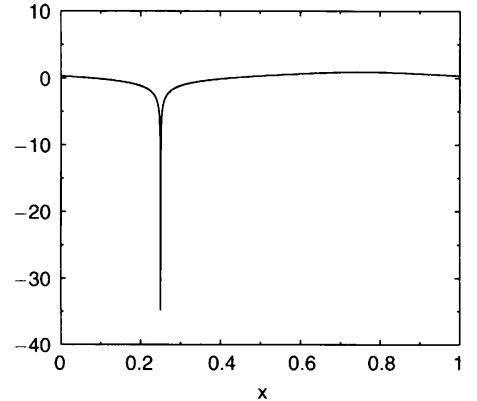

(b)
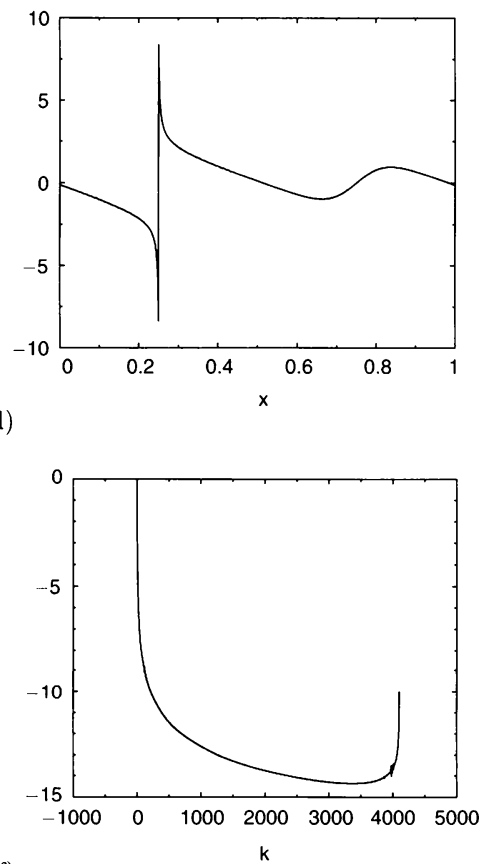

(f) 
In view of the fact that the Hilbert transform of a jump discontinuity contains a logarithmic singularity, the appearance of cusps is not surprising, but the precise nature of the singularities, and the extension of smooth solutions by weak solutions after a singularity forms, is not well understood. Moreover, it is not entirely clear that the solutions obtained by a vanishing viscosity method are physically appropriate once they develop a singularity. It is conceivable that, instead, the discontinuity "folds over," leading to some kind of multivalued solution for $\varphi$. We plan to study these questions further in later work.

The organization of this paper is as follows. In Sec. 2, we discuss Lopatinski conditions and their relationship with surface waves. In Sec. 3, we summarize the MHD equations and the jump conditions across a tangential discontinuity. In Sec. 4, we describe the small-amplitude, long-time asymptotic solution for weakly nonlinear surface waves. In Sec. 5, we solve the leading-order perturbation equations for the surface waves. This gives an eigenvalue problem for the wave velocity, and a solution for the surface wave that contains an arbitrary wave profile function, which describes the displacement of the tangential discontinuity carried by the surface wave. In Sec. 6 , we solve the second-order perturbation equations in the interior of the fluid. In Sec. 7, we use this solution in the second-order jump conditions. The imposition of solvability conditions on the resulting singular system of equations leads to the asymptotic equation (1.4)-(1.5) for the wave profile function introduced in Sec. 4. Finally, in Sec. 8, we summarize the results of the asymptotic expansion.

2. Lopatinski conditions. We consider an initial-boundary value problem for a linear, constant coefficient hyperbolic system of PDEs in a two-dimensional half-space,

$$
\begin{array}{lc}
u_{t}+A u_{x}+B u_{y}=0 & y>0, \\
C u=0 & y=0, \\
u=u_{0} & t=0 .
\end{array}
$$

Here, $u(x, y, t) \in \mathbb{R}^{m}, A, B$ are $m \times m$ matrices, and $C$ is a $p \times m$ matrix, where $p$ is the number of boundary conditions.

A basic question concerning the half-space problem (2.1)-(2.3) is what boundary conditions lead to well-posed problems. The well-posedness theory for linear, hyperbolic, half-space problems was developed by Kreiss [12] and Sakamoto [25, 26]; see [8, 18, 27] for surveys and additional references. This theory is rather subtle, and we will not discuss it in detail; here we only want to explain some of its main features to put our work in an appropriate context.

In order to study the well-posedness of (2.1)-(2.3), we look for Fourier mode solutions of (2.1) of the form

$$
u(x, y, t)=e^{i(\tau t+\xi x+\eta y)} r \quad \text { where } \tau \in \mathbb{C}^{-}, \xi \in \mathbb{R}, \eta \in \mathbb{C}^{+} .
$$

These modes grow in time, oscillate tangent to the boundary, and decay in depth. ${ }^{1}$ For given $(\tau, \xi) \in \mathbb{C}^{-} \times \mathbb{R}$, one finds that $r \in E(\tau, \xi)$, where $E(\tau, \xi)$ is a linear subspace of

\footnotetext{
${ }^{1}$ For simplicity, we assume that there are no generalized eigenvalues for $\eta$ that lead to additional polynomial factors in $y$.
} 
$\mathbb{R}^{m}$. This subspace consists of the boundary values of solutions of $(2.1)$ that are linear combinations of the Fourier modes (2.4). It can be shown that the size of the boundary matrix $C$ restricted to $E(\tau, \xi)$ does not depend on $(\tau, \xi) \in \mathbb{C}^{-} \times \mathbb{R}$, and that a necessary condition for well-posedness is that this matrix is square (meaning that $\operatorname{dim} E(\tau, \xi)=p$ ).

The Lopatinski condition for (2.1)-(2.3) states that

$$
\left.\operatorname{det} C\right|_{E(\tau, \xi)} \neq 0 \quad \text { for all }(\tau, \xi) \in \mathbb{C}^{-} \times \mathbb{R} .
$$

If this condition does not hold, then there is a nontrivial solution of the PDE (2.1) and the boundary condition (2.2) that grows in time. Since the problem is scale invariant, we may rescale this solution to obtain solutions that grow arbitrarily quickly in time, and, as a result, the problem is ill-posed with respect to any Sobolev norm. Thus, (2.5) is a necessary condition for well-posedness.

The uniform Lopatinski condition for $(2.1)-(2.3)$ states that there exists a constant $\gamma>0$ such that

$$
\left\|\left.C\right|_{E(\tau, \xi)} ^{-1}\right\| \leq \gamma \quad \text { for all }(\tau, \xi) \in \mathbb{C}^{-} \times \mathbb{R} \text { with }|\tau|^{2}+|\xi|^{2}=1 .
$$

This uniform condition is stronger than (2.5). In particular, it rules out the possibility of surface waves, which occur when

$$
\left.\operatorname{det} C\right|_{E(\tau, \xi)}=0 \quad \text { for some }(\tau, \xi) \in \mathbb{R} \times \mathbb{R} .
$$

If (2.7) holds, then (2.6) cannot hold because the matrix norm in (2.6) tends to infinity as $\operatorname{Im} \tau \rightarrow 0^{-}$.

A harmonic surface wave is a superposition of the Fourier modes (2.4) with $\tau, \xi \in \mathbb{R}$. We distinguish between genuine surface waves, in which $\eta \in \mathbb{C}^{+}$for every term in the superposition, and radiating surface waves in which at least one $\eta \in \mathbb{R}$. The genuine surface wave solutions decay exponentially away from the surface, whereas the radiating surface wave solutions do not decay and are coupled with bulk waves in the interior of the half-space.

The simplest example of this distinction is for the wave equation,

$$
u_{t t}=u_{x x}+u_{y y}
$$

Modes with "subsonic" velocity $M<1$ along the boundary $y=0$,

$$
u(x, y, t)=e^{i(x-M t)-\sqrt{1-M^{2}} y},
$$

decay away from the boundary, whereas modes with "supersonic" velocity $M>1$,

$$
u(x, y, t)=e^{i\left(x-M t \pm \sqrt{M^{2}-1} y\right)},
$$

oscillate away from the boundary. A simple example of a boundary condition for the wave equation that leads to radiating surface waves is discussed in $[18,19,27]$. A Rayleigh wave in linear elasticity is a superposition of longitudinal and transverse waves that satisfies stress-free boundary conditions. The Rayleigh wave speed is smaller than both the longitudinal and transverse wave speeds; hence the wave decays exponentially away from the surface.

The case of a subsonic surface wave, whose interior structure is determined by solving an elliptic equation, is fundamentally different from that of a supersonic wave, whose 
interior structure is determined by solving a hyperbolic equation. The ellipticity of the equations for the structure of a subsonic surface wave is related physically to the fact that, in a reference frame moving with the surface wave, a perturbation at one point of the surface can generate bulk waves that influence every other point of the surface. The appearance of the nonlocal Hilbert transform in (1.2) is directly related to the ellipticity of the interior equations for the surface wave structure, since the Hilbert transform maps the normal derivative of the boundary values of solution of Laplace's equation in a halfspace to its tangential derivative. Of course, more complicated phenomena may arise, especially in anisotropic systems; for example, we may have transonic surface waves that are subsonic with respect to some bulk waves, and supersonic with respect to others.

The well-posedness theory for half-space problems was extended to free-boundary problems for a discontinuity by Majda [16]. He related the discontinuity problem to a half-space problem by adding a new variable that describes the displacement of the discontinuity, and making a change of independent variables that "flattens" the discontinuity. The result is a system of hyperbolic PDEs that is coupled with an equation for the displacement of the discontinuity. Majda formulated analogs of the Lopatinski and uniform Lopatinski conditions for discontinuity problems, and proved [17] a short-time, nonlinear existence and stability result for Lax shocks in solutions of hyperbolic conservation laws that satisfy the uniform Lopatinski condition (see [18] and [27] for further discussion).

A general theory that encompasses the stability of Lax shocks, contact discontinuities, and non-Lax shocks in hyperbolic conservation laws, as well as the stability of discontinuities in conservation laws that are not hyperbolic, is lacking. In particular, the nonlinear evolution of discontinuities that are Lopatinski stable but not uniformly Lopatinski stable is poorly understood. One aim of this work is to study some of the phenomena that can arise in this case in the context of a concrete physical system.

3. The MHD equations. In suitably nondimensionalized variables, the incompressible MHD equations are

$$
\begin{aligned}
& \mathbf{v}_{t}+\operatorname{div}(\mathbf{v} \otimes \mathbf{v}-\mathbf{B} \otimes \mathbf{B}+p I)=0 \\
& \mathbf{B}_{t}-\operatorname{curl}(\mathbf{v} \times \mathbf{B})=0 \\
& \operatorname{div} \mathbf{v}=0
\end{aligned}
$$

Here, $\mathbf{v}$ is the velocity, $\mathbf{B}$ is the magnetic field, and $p$ is the total pressure. In addition, the magnetic field must satisfy the constraint

$$
\operatorname{div} \mathbf{B}=0,
$$

which is preserved by the evolution in time if it is satisfied by the initial data.

We consider the case of two space dimensions, and write

$$
\mathbf{v}=(u, v)^{T}, \quad \mathbf{B}=(F, G)^{T}, \quad \mathbf{u}=(u, v, F, G, p)^{T} .
$$

The MHD equations (3.1) may be written as a system of conservation laws of the form

$$
h(\mathbf{u})_{t}+f(\mathbf{u})_{x}+g(\mathbf{u})_{y}=0,
$$


where

$$
h(\mathbf{u})=\left(\begin{array}{c}
u \\
v \\
F \\
G \\
0
\end{array}\right), \quad f(\mathbf{u})=\left(\begin{array}{c}
u^{2}-F^{2}+p \\
u v-F G \\
0 \\
u G-v F \\
u
\end{array}\right), \quad g(\mathbf{u})=\left(\begin{array}{c}
u v-F G \\
v^{2}-G^{2}+p \\
v F-u G \\
0 \\
v
\end{array}\right) .
$$

The jump conditions for (3.3) across a discontinuity located at $y=\zeta(x, t)$ are

$$
\zeta_{t}[h(\mathbf{u})]+\zeta_{x}[f(\mathbf{u})]=[g(\mathbf{u})],
$$

where [.] denotes the jump across the discontinuity. In addition, the divergence free constraint,

$$
F_{x}+G_{y}=0,
$$

implies that the magnetic field must satisfy

$$
\zeta_{x}[F]=[G] .
$$

We consider wave propagation through an unperturbed state

$$
\mathbf{u}^{(0)}=\left(u_{0}, v_{0}, F_{0}, G_{0}, p_{0}\right)^{T}
$$

that consists of a stationary tangential discontinuity located at $y=0$. Thus, we have

$$
\mathbf{u}^{(0)}= \begin{cases}\mathbf{u}^{(0)-} & \text { if } y<0 \\ \mathbf{u}^{(0)+} & \text { if } y>0\end{cases}
$$

where $\mathbf{u}^{(0) \pm}=\left(u_{0}^{ \pm}, v_{0}^{ \pm}, F_{0}^{ \pm}, G_{0}^{ \pm}, p_{0}^{ \pm}\right)^{T}$ are constant states. The jump conditions (3.4) imply that

$$
\llbracket g\left(\mathbf{u}^{(0)}\right) \rrbracket=0
$$

where

$$
\llbracket g\left(\mathbf{u}^{(0)}\right) \rrbracket=g\left(\mathbf{u}^{(0)+}\right)-g\left(\mathbf{u}^{(0)-}\right) .
$$

It follows that the normal components of the velocity and magnetic field are continuous across the discontinuity. For a tangential discontinuity, these components are zero, meaning that

$$
v_{0}^{ \pm}=0, \quad G_{0}^{ \pm}=0 .
$$

The jump conditions (3.5) are then equivalent to

$$
\left[p_{0}\right]=0,
$$

so that the total pressure is constant across the discontinuity. We may assume without loss of generality that $p_{0}^{ \pm}=0$, in which case the states on either side of the discontinuity are given by

$$
\mathbf{u}^{(0) \pm}=\left(u_{0}^{ \pm}, 0, F_{0}^{ \pm}, 0,0\right)^{T} .
$$

We will consider the propagation of surface waves that are localized near the tangential discontinuity. The corresponding solutions must satisfy the decay conditions

$$
\lim _{y \rightarrow \pm \infty} \mathbf{u}(x, y, t)=\mathbf{u}^{(0) \pm} .
$$


4. The asymptotic expansion. We suppose that the perturbed tangential discontinuity has a slope of the order $\varepsilon$, where $\varepsilon$ is a small parameter. With respect to dimensionless variables in which the wavelength of the perturbation and the velocity of the surface wave are of the order one, the time scale for quadratically nonlinear effects to significantly alter the wave profile is of the order $\varepsilon^{-1}$. We therefore introduce a "slow" time variable $\tau=\varepsilon t$. We also introduce a spatial variable $\theta=x-\lambda t$ in a reference frame moving with the surface wave. Here, $\lambda$ is the linearized phase velocity of the wave, which we will determine as part of the solution (see (5.14)).

We write the perturbed location of the tangential discontinuity as

$$
y=\varepsilon \varphi(\theta, \tau ; \varepsilon)
$$

and define a new independent variable

$$
\eta=y-\varepsilon \varphi(\theta, \tau ; \varepsilon)
$$

so that the perturbed discontinuity is located at $\eta=0$. We look for an asymptotic expansion of $\mathbf{u}$ and $\varphi$ as $\varepsilon \rightarrow 0$ of the form

$$
\begin{aligned}
& \mathbf{u}^{ \pm}(\theta, \eta, \tau ; \varepsilon)=\mathbf{u}^{(0) \pm}+\varepsilon \mathbf{u}^{(1) \pm}(\theta, \eta, \tau)+\varepsilon^{2} \mathbf{u}^{(2) \pm}(\theta, \eta, \tau)+O\left(\varepsilon^{3}\right), \\
& \varphi(\theta, \tau ; \varepsilon)=\varphi^{(1)}(\theta, \tau)+\varepsilon \varphi^{(2)}(\theta, \tau)+O\left(\varepsilon^{2}\right),
\end{aligned}
$$

where $\mathbf{u}=\mathbf{u}^{+}$in $\eta>0$ and $\mathbf{u}=\mathbf{u}^{-}$in $\eta<0$.

Using the method of multiple scales, we expand the partial derivatives with respect to the original time and space variables as

$$
\begin{aligned}
& \partial_{t}=-\lambda \partial_{\theta}+\varepsilon\left(\partial_{\tau}+\lambda \varphi_{\theta} \partial_{\eta}\right)-\varepsilon^{2} \varphi_{\tau} \partial_{\eta} \\
& \partial_{x}=\partial_{\theta}-\varepsilon \varphi_{\theta} \partial_{\eta} \\
& \partial_{y}=\partial_{\eta}
\end{aligned}
$$

We use these expansions in (3.3), Taylor expand the result with respect to $\varepsilon$, and equate coefficients of $\varepsilon^{0}$ and $\varepsilon^{1}$ to zero. Introducing the matrices

$$
A^{ \pm}=\nabla f\left(\mathbf{u}^{(0) \pm}\right), \quad B^{ \pm}=\nabla g\left(\mathbf{u}^{(0) \pm}\right), \quad C^{ \pm}=\nabla h\left(\mathbf{u}^{(0) \pm}\right),
$$

we find that, in the interior, the asymptotic solution satisfies

$$
\begin{aligned}
&(A-\lambda C) \mathbf{u}_{\theta}^{(1)}+B \mathbf{u}_{\eta}^{(1)}=0 \\
&(A-\lambda C) \mathbf{u}_{\theta}^{(2)}+B \mathbf{u}_{\eta}^{(2)}=-\left\{C \mathbf{u}_{\tau}^{(1)}-\varphi_{\theta}^{(1)}(A-\lambda C) \mathbf{u}_{\eta}^{(1)}\right. \\
&\left.+\frac{1}{2} \nabla^{2}(f-\lambda h) \cdot\left(\mathbf{u}^{(1)}, \mathbf{u}^{(1)}\right)_{\theta}+\frac{1}{2} \nabla^{2} g \cdot\left(\mathbf{u}^{(1)}, \mathbf{u}^{(1)}\right)_{\eta}\right\}
\end{aligned}
$$

where we drop the \pm -superscripts to simplify the notation.

Expanding the jump condition in (3.4), with $\zeta=\varepsilon \varphi$, and equating coefficients of $\varepsilon$ and $\varepsilon^{2}$, we find that the solutions of the previous equations satisfy the following jump 
conditions on $\eta=0$ :

$$
\begin{aligned}
& \llbracket B \mathbf{u}^{(1)} \rrbracket-\varphi_{\theta}^{(1)} \llbracket f\left(\mathbf{u}^{(0)}\right)-\lambda h\left(\mathbf{u}^{(0)}\right) \rrbracket=0, \\
& \llbracket B \mathbf{u}^{(2)} \rrbracket-\varphi_{\theta}^{(2)} \llbracket f\left(\mathbf{u}^{(0)}\right)-\lambda h\left(\mathbf{u}^{(0)}\right) \rrbracket=\varphi_{\tau}^{(1)} \llbracket h\left(\mathbf{u}^{(0)}\right) \rrbracket \\
& \quad+\varphi_{\theta}^{(1)} \llbracket(A-\lambda C) \mathbf{u}^{(1)} \rrbracket-\frac{1}{2} \llbracket \nabla^{2} g \cdot\left(\mathbf{u}^{(1)}, \mathbf{u}^{(1)}\right) \rrbracket .
\end{aligned}
$$

In the rest of this paper, we solve equations (4.1)-(4.4).

5. The first-order equations. The first-order interior equations (4.1), in $\eta>0$ and $\eta<0$, are

$$
\left(A^{ \pm}-\lambda C^{ \pm}\right) \mathbf{u}_{\theta}^{(1) \pm}+B^{ \pm} \mathbf{u}_{\eta}^{(1) \pm}=0
$$

The first-order jump conditions (4.3) are

$$
\llbracket B \mathbf{u}^{(1)} \rrbracket-\varphi_{\theta}^{(1)} \llbracket f\left(\mathbf{u}^{(0)}\right)-\lambda h\left(\mathbf{u}^{(0)}\right) \rrbracket=0
$$

Introducing the Fourier transforms

$$
\begin{aligned}
\mathbf{U}^{(1) \pm}(k, \eta, \tau) & =\frac{1}{2 \pi} \int_{-\infty}^{+\infty} \mathbf{u}^{(1) \pm}(\theta, \eta, \tau) e^{-i k \theta} d \theta \\
\psi^{(1)}(k, \tau) & =\frac{1}{2 \pi} \int_{-\infty}^{+\infty} \varphi^{(1)}(\theta, \tau) e^{-i k \theta} d \theta
\end{aligned}
$$

and Fourier transforming (5.1)-(5.2) with respect to $\theta$, we find that

$$
\begin{aligned}
& i k\left(A^{ \pm}-\lambda C^{ \pm}\right) \mathbf{U}^{(1) \pm}+B^{ \pm} \mathbf{U}_{\eta}^{(1) \pm}=0, \\
& \llbracket B \mathbf{U}^{(1)} \rrbracket-i k \psi^{(1)} \llbracket f\left(\mathbf{u}^{(0)}\right)-\lambda h\left(\mathbf{u}^{(0)}\right) \rrbracket=0 .
\end{aligned}
$$

The boundary conditions (3.7) imply that

$$
\lim _{\eta \rightarrow \pm \infty} \mathbf{U}^{(1) \pm}(k, \eta, \tau)=0 .
$$

The general solution of (5.3) is

$$
\mathbf{U}^{(1) \pm}(k, \eta, \tau)=a^{ \pm}(k, \tau) e^{-k \eta} \mathbf{R}^{ \pm}+b^{ \pm}(k, \tau) e^{k \eta} \overline{\mathbf{R}}^{ \pm},
$$

where $a^{ \pm}(k, \tau)$ and $b^{ \pm}(k, \tau)$ are arbitrary complex-valued functions, the bar denotes a complex conjugate, and the eigenvector $\mathbf{R}^{ \pm}$satisfies

$$
\left[i\left(A^{ \pm}-\lambda C^{ \pm}\right)-B^{ \pm}\right] \mathbf{R}^{ \pm}=0 .
$$

After a convenient choice of normalization, this eigenvector is given explicitly by

$$
\mathbf{R}^{ \pm}=\left(c^{ \pm}, i c^{ \pm},-F_{0}^{ \pm},-i F_{0}^{ \pm},\left(c^{ \pm}\right)^{2}-\left(F_{0}^{ \pm}\right)^{2}\right)^{T},
$$

where

$$
c^{ \pm}=\lambda-u_{0}^{ \pm}
$$


Imposing the condition in (5.5) on (5.6), we find that

$$
\begin{aligned}
& \mathbf{U}^{(1)+}(k, \eta, \tau)= \begin{cases}b^{+}(k, \tau) e^{k \eta} \overline{\mathbf{R}}^{+}, & \text {if } k<0, \\
a^{+}(k, \tau) e^{-k \eta} \mathbf{R}^{+}, & \text {if } k>0,\end{cases} \\
& \mathbf{U}^{(1)-}(k, \eta, \tau)= \begin{cases}a^{-}(k, \tau) e^{-k \eta} \mathbf{R}^{-}, & \text {if } k<0, \\
b^{-}(k, \tau) e^{k \eta} \overline{\mathbf{R}}^{-}, & \text {if } k>0 .\end{cases}
\end{aligned}
$$

Next, we use the solution (5.10)-(5.11) in the jump condition (5.4). First, we consider the case $k>0$. The resulting equations may be written as a system of linear equations for the unknowns $\left(a^{+}, b^{-}, k \psi^{(1)}\right)$ :

$$
\left(B^{+} \mathbf{R}^{+},-B^{-} \overline{\mathbf{R}}^{-},-i \llbracket f\left(\mathbf{u}^{(0)}\right)-\lambda h\left(\mathbf{u}^{(0)}\right) \rrbracket\right)\left(\begin{array}{c}
a^{+} \\
b^{-} \\
k \psi^{(1)}
\end{array}\right)=0 .
$$

After some algebra, we find that this system is equivalent to

$$
\left(\begin{array}{ccc}
F_{0}^{+} F_{0}^{-}-c^{+} c^{-} & F_{0}^{+} F_{0}^{-}-c^{+} c^{-} & 0 \\
\left(c^{+}\right)^{2}-\left(F_{0}^{+}\right)^{2} & -\left(c^{-}\right)^{2}+\left(F_{0}^{-}\right)^{2} & 0 \\
F_{0}^{+} & F_{0}^{-} & F_{0}^{+}-F_{0}^{-} \\
c^{+} & c^{-} & -u_{0}^{+}+u_{0}^{-}
\end{array}\right)\left(\begin{array}{c}
a^{+} \\
b^{-} \\
k \psi^{(1)}
\end{array}\right)=0 .
$$

This system has a nontrivial solution if

$$
\left(c^{+}\right)^{2}+\left(c^{-}\right)^{2}=\left(F_{0}^{+}\right)^{2}+\left(F_{0}^{-}\right)^{2} .
$$

Using (5.9) in (5.13) and simplifying the result, we get a quadratic equation for the wave velocity $\lambda$,

$$
2 \lambda^{2}-2\left(u_{0}^{+}+u_{0}^{-}\right) \lambda+\left(u_{0}^{+}\right)^{2}+\left(u_{0}^{-}\right)^{2}=\left(F_{0}^{+}\right)^{2}+\left(F_{0}^{-}\right)^{2} .
$$

This equation has two distinct real roots for $\lambda$ if

$$
\frac{1}{2}\left(u_{0}^{+}-u_{0}^{-}\right)^{2}<\left(F_{0}^{+}\right)^{2}+\left(F_{0}^{+}\right)^{2} .
$$

This condition is equivalent to the stability condition in (1.1). We assume that (5.15) holds, and choose $\lambda$ to be one of the roots of (5.14). The solution of (5.12) is then

$$
a^{+}=-k \psi^{(1)}, \quad b^{-}=k \psi^{(1)} .
$$

For $k<0$, we proceed in a similar way, solving an algebraic system for the unknowns $\left(b^{+}, a^{-}, k \psi^{(1)}\right)$ :

$$
\left(B^{+} \overline{\mathbf{R}}^{+},-B^{-} \mathbf{R}^{-},-i \llbracket f\left(\mathbf{u}^{(0)}\right)-\lambda h\left(\mathbf{u}^{(0)}\right) \rrbracket\right)\left(\begin{array}{c}
b^{+} \\
a^{-} \\
k \psi^{(1)}
\end{array}\right)=0 .
$$

As before, this system has a nontrivial solution if $\lambda$ is a root of (5.14). The solution is given by

$$
b^{+}=k \psi^{(1)}, \quad a^{-}=-k \psi^{(1)} .
$$


Summarizing these results, we have shown that when $\lambda$ satisfies (5.14), the solution of (5.3)-(5.5) is given by

$$
\begin{aligned}
& \mathbf{U}^{(1)-}(k, \eta, \tau)= \begin{cases}-k \psi^{(1)}(k, \tau) e^{-k \eta} \mathbf{R}^{-}, & \text {if } k<0, \\
k \psi^{(1)}(k, \tau) e^{k \eta} \overline{\mathbf{R}}^{-}, & \text {if } k>0,\end{cases} \\
& \mathbf{U}^{(1)+}(k, \eta, \tau)= \begin{cases}k \psi^{(1)}(k, \tau) e^{k \eta} \overline{\mathbf{R}}^{+}, & \text {if } k<0, \\
-k \psi^{(1)}(k, \tau) e^{-k \eta} \mathbf{R}^{+}, & \text {if } k>0,\end{cases}
\end{aligned}
$$

where $\psi^{(1)}(k, \tau)$ is an arbitrary function of integration. We may write this solution in a more compact form as

$$
\mathbf{U}^{(1) \pm}(k, \eta, \tau)=\mp|k| \psi^{(1)}(k, \tau) e^{\mp|k| \eta} \operatorname{Re} \mathbf{R}^{ \pm}-i k \psi^{(1)}(k, \tau) e^{\mp|k| \eta} \operatorname{Im} \mathbf{R}^{ \pm} .
$$

Here, $\operatorname{Re} \mathbf{R}^{ \pm}$and $\operatorname{Im} \mathbf{R}^{ \pm}$denote the real and imaginary parts of $\mathbf{R}^{ \pm}$, respectively. Explicitly, from (5.8), we have

$$
\begin{aligned}
\operatorname{Re} \mathbf{R}^{ \pm} & =\left(c^{ \pm}, 0,-F_{0}^{ \pm}, 0,\left(c^{ \pm}\right)^{2}-\left(F_{0}^{ \pm}\right)^{2}\right)^{T}, \\
\operatorname{Im} \mathbf{R}^{ \pm} & =\left(0, c^{ \pm}, 0,-F_{0}^{ \pm}, 0\right)^{T} .
\end{aligned}
$$

Inverting the Fourier transform, we find that the first-order solution is given by

$$
\begin{aligned}
\mathbf{u}^{(1) \pm}(\theta, \eta, \tau) & =\int_{-\infty}^{+\infty} \mathbf{U}^{(1) \pm}(k, \eta, \tau) e^{i k \theta} d k \\
& =\mp \rho^{ \pm}(\theta, \eta, \tau) \operatorname{Re} \mathbf{R}^{ \pm}-i \chi^{ \pm}(\theta, \eta, \tau) \operatorname{Im} \mathbf{R}^{ \pm}
\end{aligned}
$$

where

$$
\begin{aligned}
\rho^{ \pm}(\theta, \eta, \tau) & =\int_{-\infty}^{+\infty}|k| \psi^{(1)}(k, \tau) e^{i k \theta \mp|k| \eta} d k, \\
\chi^{ \pm}(\theta, \eta, \tau) & =\int_{-\infty}^{+\infty} k \psi^{(1)}(k, \tau) e^{i k \theta \mp|k| \eta} d k .
\end{aligned}
$$

This solution depends on the arbitrary function $\psi^{(1)}(k, \tau)$, which describes the profile of the surface wave. In the next two sections, we derive an evolution equation for this function by imposing solvability conditions on the equations for the second-order corrections to this first-order solution.

6. The second-order interior equations. The second-order interior equations (4.2) are

$$
(A-\lambda C) \mathbf{u}_{\theta}^{(2)}+B \mathbf{u}_{\eta}^{(2)}=\mathbf{p}
$$

where

$$
\begin{aligned}
\mathbf{p}=- & \left\{C \mathbf{u}_{\tau}^{(1)}-\varphi_{\theta}^{(1)}(A-\lambda C) \mathbf{u}_{\eta}^{(1)}\right. \\
& \left.+\frac{1}{2} \nabla^{2}(f-\lambda h) \cdot\left(\mathbf{u}^{(1)}, \mathbf{u}^{(1)}\right)_{\theta}+\frac{1}{2} \nabla^{2} g \cdot\left(\mathbf{u}^{(1)}, \mathbf{u}^{(1)}\right)_{\eta}\right\} .
\end{aligned}
$$


Introducing the Fourier transforms

$$
\begin{aligned}
\mathbf{U}^{(2)}(k, \eta, \tau) & =\frac{1}{2 \pi} \int_{-\infty}^{+\infty} \mathbf{u}^{(2)}(\theta, \eta, \tau) e^{-i k \theta} d \theta \\
\mathbf{P}(k, \eta, \tau) & =\frac{1}{2 \pi} \int_{-\infty}^{+\infty} \mathbf{p}(\theta, \eta, \tau) e^{-i k \theta} d \theta
\end{aligned}
$$

and Fourier transforming (6.1) with respect to $\theta$, we get

$$
i k(A-\lambda C) \mathbf{U}^{(2)}+B \mathbf{U}_{\eta}^{(2)}=\mathbf{P} .
$$

Restoring the \pm -superscripts for a moment, we see from (3.7) and (6.3) that the solution of (6.5) must satisfy the decay condition

$$
\lim _{\eta \rightarrow \pm \infty} \mathbf{U}^{(2) \pm}(k, \eta, \tau)=0 .
$$

In order to solve (6.5), we introduce a left eigenvector $\mathbf{L}$ such that

$$
\mathbf{L} \cdot[i(A-\lambda C)-B]=0 .
$$

We normalize $\mathbf{L}$ so that

$$
\mathbf{L} \cdot B \mathbf{R}=\overline{\mathbf{L}} \cdot B \overline{\mathbf{R}}=1 .
$$

It follows from the equations satisfied by $\mathbf{L}$ and $\mathbf{R}$ that

$$
\mathbf{L} \cdot B \overline{\mathbf{R}}=\overline{\mathbf{L}} \cdot B \mathbf{R}=0 .
$$

Explicitly, we have

$$
\mathbf{L}=\frac{1}{2\left(c^{2}-F_{0}^{2}\right)}\left(-i, 1, \frac{i F_{0}}{\lambda},-\frac{F_{0}}{\lambda},-i\left(c-u_{0}\right)\right)^{T} .
$$

We also introduce a linear subspace consisting of the vectors $\mathbf{S}$ such that

$$
\mathbf{L} \cdot B \mathbf{S}=\overline{\mathbf{L}} \cdot B \mathbf{S}=0 .
$$

This subspace is complementary to the subspace spanned by $\{\mathbf{R}, \overline{\mathbf{R}}\}$.

We write the solution of $(6.5)$ in the form

$$
\mathbf{U}^{(2)}(k, \eta, \tau)=\mathbf{S}(k, \eta, \tau)+a(k, \eta, \tau) \mathbf{R}+b(k, \eta, \tau) \overline{\mathbf{R}}
$$

where $\mathbf{S}$ satisfies (6.9). We will solve for the vector-valued function $\mathbf{S}$ and the scalarvalued functions $a, b$. Using (6.10) in (6.5), and rewriting the result with the help of (5.7), we find that

$$
i k(A-\lambda C) \mathbf{S}+B \mathbf{S}_{\eta}+\left(a_{\eta}+k a\right) B \mathbf{R}+\left(b_{\eta}-k b\right) B \overline{\mathbf{R}}=\mathbf{P} .
$$

First, we solve (6.11) for $a$ and $b$. Left multiplying (6.11) by $\mathbf{L}$ and $\overline{\mathbf{L}}$, and using (6.8) and (6.9), we find that

$$
\begin{aligned}
a_{\eta}+k a & =\mathbf{L} \cdot \mathbf{P} \\
b_{\eta}-k b & =\overline{\mathbf{L}} \cdot \mathbf{P} .
\end{aligned}
$$


The solutions of these equations are

$$
\begin{aligned}
& a(k, \eta, \tau)=e^{-k \eta}\left(a_{0}(k, \tau)+\int_{0}^{\eta} \mathbf{L} \cdot \mathbf{P}\left(k, \eta^{\prime}, \tau\right) e^{k \eta^{\prime}} d \eta^{\prime}\right) \\
& b(k, \eta, \tau)=e^{k \eta}\left(b_{0}(k, \tau)+\int_{0}^{\eta} \overline{\mathbf{L}} \cdot \mathbf{P}\left(k, \eta^{\prime}, \tau\right) e^{-k \eta^{\prime}} d \eta^{\prime}\right)
\end{aligned}
$$

where $a_{0}(k, \tau), b_{0}(k, \tau)$ are arbitrary functions of integration.

Next, we solve (6.11) for S. Introducing a convenient basis of the complementary space defined by $(6.9)$, we write

$$
\mathbf{S}=\left(s_{1}, s_{2}, s_{3}, \frac{\lambda c-2 F_{0}^{2}}{F_{0}\left(c-u_{0}\right)} s_{2}, 0\right)^{T}
$$

where the functions $s_{1}, s_{2}, s_{3}$ are the components of $\mathbf{S}$. We let $\mathbf{L}_{j}$, with $j=1,2,3$, be vectors such that

$$
\mathbf{L}_{j} \cdot B \mathbf{R}=\mathbf{L}_{j} \cdot B \overline{\mathbf{R}}=0, \quad i \mathbf{L}_{j} \cdot(A-\lambda C) \mathbf{S}=s_{j}
$$

They are given explicitly by

$$
\begin{aligned}
& \mathbf{L}_{1}=\frac{i}{c^{2}-F_{0}^{2}}\left(c, 0,-\frac{2 c F_{0}}{\lambda}, 0, F_{0}^{2}-c u_{0}\right)^{T}, \\
& \mathbf{L}_{2}=\left(0,0,0, \frac{i F_{0}\left(c-u_{0}\right)}{\lambda\left(c^{2}-F_{0}^{2}\right)}, 0\right)^{T}, \\
& \mathbf{L}_{3}=\frac{i}{c^{2}-F_{0}^{2}}\left(-F_{0}, 0,-\frac{c^{2}+F_{0}^{2}}{\lambda}, 0,-F_{0}\left(c-u_{0}\right)\right)^{T} .
\end{aligned}
$$

Left multiplying (6.11) by $\mathbf{L}_{j}$, and using (6.15) in the result, we obtain that

$$
\begin{aligned}
& k s_{1}-i s_{2 \eta}=\mathbf{L}_{1} \cdot \mathbf{P}, \\
& k s_{2}=\mathbf{L}_{2} \cdot \mathbf{P} \\
& k s_{3}-i \frac{F_{0}^{2}-c u_{0}}{F_{0}\left(c-u_{0}\right)} s_{2 \eta}=\mathbf{L}_{3} \cdot \mathbf{P} .
\end{aligned}
$$

The solution of these equations is

$$
\begin{aligned}
s_{1} & =\frac{1}{k} \mathbf{L}_{1} \cdot \mathbf{P}+\frac{i}{k^{2}} \mathbf{L}_{2} \cdot \mathbf{P}_{\eta}, \\
s_{2} & =\frac{1}{k} \mathbf{L}_{2} \cdot \mathbf{P} \\
s_{3} & =\frac{1}{k} \mathbf{L}_{3} \cdot \mathbf{P}+\frac{i}{k^{2}} \frac{F_{0}^{2}-c u_{0}}{F_{0}\left(c-u_{0}\right)} \mathbf{L}_{2} \cdot \mathbf{P}_{\eta} .
\end{aligned}
$$

The solution for $\mathbf{S}$ is then given by (6.14) and (6.16). 
To write out explicit expressions for the terms in this solution, it is convenient to restore the \pm -superscripts. Using (5.16) in (6.2), we find after some algebra that

$$
\mathbf{p}^{ \pm}=\left(\begin{array}{l} 
\pm c^{ \pm} \rho_{\tau}^{ \pm} \mp\left(\left(F_{0}^{ \pm}\right)^{2}+c^{ \pm} u_{0}^{ \pm}\right) \varphi_{\theta}^{(1)} \rho_{\eta}^{ \pm} \\
-\left(\left(c^{ \pm}\right)^{2}-\left(F_{0}^{ \pm}\right)^{2}\right)\left[\left(\left(\rho^{ \pm}\right)^{2}\right)_{\theta} \pm i\left(\chi^{ \pm} \rho^{ \pm}\right)_{\eta}\right] \\
i c^{ \pm} \chi_{\tau}^{ \pm}+i\left(\left(c^{ \pm}\right)^{2}-\left(F_{0}^{ \pm}\right)^{2}\right) \varphi_{\theta}^{(1)} \chi_{\eta}^{ \pm} \\
\mp i\left(\left(c^{ \pm}\right)^{2}-\left(F_{0}^{ \pm}\right)^{2}\right)\left[\left(\chi^{ \pm} \rho^{ \pm}\right)_{\theta} \pm i\left(\left(\chi^{ \pm}\right)^{2}\right)_{\eta}\right] \\
\mp F_{0}^{ \pm} \rho_{\tau}^{ \pm} \mp \lambda F_{0}^{ \pm} \varphi_{\theta}^{(1)} \rho_{\eta}^{ \pm} \\
-i F_{0}^{ \pm} \chi_{\tau}^{ \pm} \\
\mp c^{ \pm} \varphi_{\theta}^{(1)} \rho_{\eta}^{ \pm}
\end{array}\right)
$$

In order to compute the Fourier transform $\mathbf{P}^{ \pm}$of $\mathbf{p}^{ \pm}$, we use the following equations which follow from (5.17)-(5.18):

$$
\begin{aligned}
& \frac{1}{2 \pi} \int_{-\infty}^{+\infty} \chi_{\tau}^{ \pm} e^{-i k \theta} d \theta=k \psi_{\tau}^{(1)} e^{\mp|k| \eta}, \\
& \frac{1}{2 \pi} \int_{-\infty}^{+\infty} \rho_{\tau}^{ \pm} e^{-i k \theta} d \theta=|k| \psi_{\tau}^{(1)} e^{\mp|k| \eta}, \\
& \frac{1}{2 \pi} \int_{-\infty}^{+\infty} \varphi_{\theta}^{(1)} \chi_{\eta}^{ \pm} e^{-i k \theta} d \theta=\mp i \int_{-\infty}^{+\infty}(k-l) l|l| \psi^{(1)}(k-l) \psi^{(1)}(l) e^{\mp|l| \eta} d l, \\
& \frac{1}{2 \pi} \int_{-\infty}^{+\infty} \varphi_{\theta}^{(1)} \rho_{\eta}^{ \pm} e^{-i k \theta} d \theta=\mp i \int_{-\infty}^{+\infty}(k-l) l^{2} \psi^{(1)}(k-l) \psi^{(1)}(l) e^{\mp|l| \eta} d l, \\
& \frac{1}{2 \pi} \int_{-\infty}^{+\infty}\left[\left(\left(\rho^{ \pm}\right)^{2}\right)_{\theta} \pm i\left(\chi^{ \pm} \rho^{ \pm}\right)_{\eta}\right] e^{-i k \theta} d \theta \\
& \quad=i \int_{-\infty}^{+\infty}(|k-l||l|-(k-l) l)|l| \psi^{(1)}(k-l) \psi^{(1)}(l) e^{\mp(|k-l|+|l|) \eta} d l, \\
& \frac{1}{2 \pi} \int_{-\infty}^{+\infty}\left[\left(\chi^{ \pm} \rho^{ \pm}\right)_{\theta} \pm i\left(\left(\chi^{ \pm}\right)^{2}\right)_{\eta}\right] e^{-i k \theta} d \theta \\
& \quad=i \int_{-\infty}^{+\infty}(|k-l| l-(k-l)|l|) l \psi^{(1)}(k-l) \psi^{(1)}(l) e^{\mp(|k-l|+|l|) \eta} d l .
\end{aligned}
$$

Here, we do not show explicitly the dependence of functions on the slow time variable $\tau$ to simplify the notation.

We use (6.17) in (6.4), and simplify the result with the help of these equations. Using the resulting expression for $\mathbf{P}^{ \pm}$in $(6.12),(6.13)$ and $(6.16)$, we obtain after some algebra 
that

$$
\begin{aligned}
& a^{ \pm}=e^{-k \eta}\left\{a_{0}^{ \pm}+i \frac{\lambda c^{ \pm}+2\left(F_{0}^{ \pm}\right)^{2}}{2 \lambda\left(\left(c^{ \pm}\right)^{2}-\left(F_{0}^{ \pm}\right)^{2}\right)}\left(e^{(k \mp|k|) \eta}-1\right) \psi_{\tau}^{(1)}\right. \\
& \pm \int_{0}^{ \pm \infty} l^{2} \psi^{(1)}(k-l) \psi^{(1)}(l)\left(e^{(k-l) \eta}-1\right) d l \\
& \left. \pm \int_{\mp \infty}^{0} \frac{1}{2}(k-l \pm|k-l|) l \psi^{(1)}(k-l) \psi^{(1)}(l)\left(e^{2 l \eta}-1\right) d l\right\}, \\
& b^{ \pm}=e^{k \eta}\left\{b_{0}^{ \pm}-i \frac{\lambda c^{ \pm}+2\left(F_{0}^{ \pm}\right)^{2}}{2 \lambda\left(\left(c^{ \pm}\right)^{2}-\left(F_{0}^{ \pm}\right)^{2}\right)}\left(e^{-(k \pm|k|) \eta}-1\right) \psi_{\tau}^{(1)}\right. \\
& \pm \int_{\mp \infty}^{0} l^{2} \psi^{(1)}(k-l) \psi^{(1)}(l)\left(e^{-(k-l) \eta}-1\right) d l \\
& \left. \pm \int_{0}^{ \pm \infty} \frac{1}{2}(k-l \mp|k-l|) l \psi^{(1)}(k-l) \psi^{(1)}(l)\left(e^{-2 l \eta}-1\right) d l\right\}, \\
& s_{1}^{ \pm}= \pm i \frac{\left(c^{ \pm}\right)^{2}+\left(F_{0}^{ \pm}\right)^{2}}{\left(c^{ \pm}\right)^{2}-\left(F_{0}^{ \pm}\right)^{2}} \frac{|k|}{k} e^{\mp|k| \eta} \psi_{\tau}^{(1)} \\
& -\frac{c^{ \pm}}{k} \int_{-\infty}^{+\infty}((k-l) l-|k-l||l|) l \psi^{(1)}(k-l) \psi^{(1)}(l) e^{\mp(|k-l|+|l|) \eta} d l \\
& s_{2}^{ \pm}=\frac{\left(F_{0}^{ \pm}\right)^{2}\left(c^{ \pm}-u_{0}^{ \pm}\right)}{\lambda\left(\left(c^{ \pm}\right)^{2}-\left(F_{0}^{ \pm}\right)^{2}\right)} e^{\mp|k| \eta} \psi_{\tau}^{(1)}, \\
& s_{3}^{ \pm}=\mp i \frac{2 c^{ \pm} F_{0}^{ \pm}}{\left(c^{ \pm}\right)^{2}-\left(F_{0}^{ \pm}\right)^{2}} \frac{|k|}{k} e^{\mp|k| \eta} \psi_{\tau}^{(1)} \\
& +\frac{F_{0}^{ \pm}}{k} \int_{-\infty}^{+\infty}((k-l) l-|k-l||l|) l \psi^{(1)}(k-l) \psi^{(1)}(l) e^{\mp(|k-l|+|l|) \eta} d l .
\end{aligned}
$$

Thus, the Fourier transform (6.3) of the solution of the second-order interior equations $(6.1)-(6.2)$ is given by (6.10) and (6.14), where the coefficients are given by (6.18)-(6.22).

The function $\mathbf{S}^{ \pm}$depends on $\eta$ only through the exponentials of $\mp|k| \eta$ and $\mp(|k-l|+|l|) \eta$, so that

$$
\lim _{\eta \rightarrow \pm \infty} \mathbf{S}^{ \pm}(k, \eta, \tau)=0
$$

Thus, the function $\mathbf{U}^{(2) \pm}$ in (6.10) satisfies the decay condition (6.6) if and only if

$$
\begin{aligned}
& \lim _{\eta \rightarrow \pm \infty} a^{ \pm}(k, \eta, \tau)=0, \\
& \lim _{\eta \rightarrow \pm \infty} b^{ \pm}(k, \eta, \tau)=0 .
\end{aligned}
$$

If $k<0$, the conditions (6.23) for $a^{-}$and (6.24) for $b^{+}$are automatically satisfied, while the conditions (6.23) for $a^{+}$and (6.24) for $b^{-}$determine the functions $a_{0}^{+}$and $b_{0}^{-}$, 
respectively. The result is that

$$
\begin{aligned}
a_{0}^{+}= & i \frac{\lambda c^{+}+2\left(F_{0}^{+}\right)^{2}}{2 \lambda\left(\left(c^{+}\right)^{2}-\left(F_{0}^{+}\right)^{2}\right)} \psi_{\tau}^{(1)}+\int_{0}^{+\infty} l^{2} \psi^{(1)}(k-l) \psi^{(1)}(l) d l \\
& +\int_{-\infty}^{0} \frac{1}{2}(k-l+|k-l|) l \psi^{(1)}(k-l) \psi^{(1)}(l) d l \\
b_{0}^{-}= & -i \frac{\lambda c^{-}+2\left(F_{0}^{-}\right)^{2}}{2 \lambda\left(\left(c^{-}\right)^{2}-\left(F_{0}^{-}\right)^{2}\right)} \psi_{\tau}^{(1)}+\int_{0}^{+\infty} l^{2} \psi^{(1)}(k-l) \psi^{(1)}(l) d l \\
& +\int_{-\infty}^{0} \frac{1}{2}(k-l+|k-l|) l \psi^{(1)}(k-l) \psi^{(1)}(l) d l .
\end{aligned}
$$

In a similar way, if $k>0$, the conditions (6.23) for $a^{-}$and (6.24) for $b^{+}$determine $a_{0}^{-}$ and $b_{0}^{+}$, with the result that

$$
\begin{aligned}
a_{0}^{-}= & i \frac{\lambda c^{-}+2\left(F_{0}^{-}\right)^{2}}{2 \lambda\left(\left(c^{-}\right)^{2}-\left(F_{0}^{-}\right)^{2}\right)} \psi_{\tau}^{(1)}+\int_{-\infty}^{0} l^{2} \psi^{(1)}(k-l) \psi^{(1)}(l) d l \\
& +\int_{0}^{+\infty} \frac{1}{2}(k-l-|k-l|) l \psi^{(1)}(k-l) \psi^{(1)}(l) d l \\
b_{0}^{+}= & -i \frac{\lambda c^{+}+2\left(F_{0}^{+}\right)^{2}}{2 \lambda\left(\left(c^{+}\right)^{2}-\left(F_{0}^{+}\right)^{2}\right)} \psi_{\tau}^{(1)}+\int_{-\infty}^{0} l^{2} \psi^{(1)}(k-l) \psi^{(1)}(l) d l \\
& +\int_{0}^{+\infty} \frac{1}{2}(k-l-|k-l|) l \psi^{(1)}(k-l) \psi^{(1)}(l) d l .
\end{aligned}
$$

After some algebra, we may write the corresponding solutions for $a^{ \pm}$and $b^{ \pm}$as

$$
\begin{aligned}
& a^{-}= \begin{cases}e^{-k \eta}\left[a_{0}^{-}+\int_{-\infty}^{+\infty} \Omega_{0}^{\prime} \psi^{(1)}(k-l) \psi^{(1)}(l) d l\right] & \text { if } k<0, \\
i \alpha^{-} e^{k \eta} \psi_{\tau}^{(1)}+\int_{-\infty}^{0} \Omega \psi^{(1)}(k-l) \psi^{(1)}(l) d l & \text { if } k>0,\end{cases} \\
& a^{+}= \begin{cases}i \alpha^{+} e^{k \eta} \psi_{\tau}^{(1)}+\int_{0}^{+\infty} \Omega \psi^{(1)}(k-l) \psi^{(1)}(l) d l & \text { if } k<0, \\
e^{-k \eta}\left[a_{0}^{+}+\int_{-\infty}^{+\infty} \Omega_{0} \psi^{(1)}(k-l) \psi^{(1)}(l) d l\right] & \text { if } k>0,\end{cases} \\
& b^{-}= \begin{cases}-i \alpha^{-} e^{-k \eta} \psi_{\tau}^{(1)}+\int_{0}^{+\infty} \Omega \psi^{(1)}(k-l) \psi^{(1)}(l) d l & \text { if } k<0, \\
e^{k \eta}\left[b_{0}^{-}+\int_{-\infty}^{+\infty} \Omega_{0} \psi^{(1)}(k-l) \psi^{(1)}(l) d l\right] & \text { if } k>0,\end{cases} \\
& b^{+}= \begin{cases}e^{k \eta}\left[b_{0}^{+}+\int_{-\infty}^{+\infty} \Omega_{0}^{\prime} \psi^{(1)}(k-l) \psi^{(1)}(l) d l\right] \\
-i \alpha^{+} e^{-k \eta} \psi_{\tau}^{(1)}+\int_{-\infty}^{0} \Omega \psi^{(1)}(k-l) \psi^{(1)}(l) d l & \text { if } k>0,\end{cases}
\end{aligned}
$$

where

$$
\alpha^{ \pm}=\frac{\lambda c^{ \pm}+2\left(F_{0}^{ \pm}\right)^{2}}{2 \lambda\left(\left(c^{ \pm}\right)^{2}-\left(F_{0}^{ \pm}\right)^{2}\right)},
$$

and

$$
\begin{aligned}
\Omega(k, l, \eta) & =\left[l+(k-l) e^{-|(l-k) \eta|}\right] l e^{-|l \eta|} \\
\Omega_{0}(k, l, \eta) & =\left[l^{2}\left(e^{-(l-k)|\eta|}-1\right)\right] \frac{l+|l|}{2 l}+\left[(k-l) l\left(e^{2 l|\eta|}-1\right)\right] \frac{l-|l|}{2 l}, \\
\Omega_{0}^{\prime}(k, l, \eta) & =\left[l^{2}\left(e^{(l-k)|\eta|}-1\right)\right] \frac{l-|l|}{2 l}+\left[(k-l) l\left(e^{-2 l|\eta|}-1\right)\right] \frac{l+|l|}{2 l} .
\end{aligned}
$$


Summarizing the results of this section, we have shown that the Fourier transform (6.3) of the solution of the second-order interior equations (6.1)-(6.2) and (6.6) is given by (6.10) and (6.14), where the coefficients $s_{1}^{ \pm}, s_{2}^{ \pm}, s_{3}^{ \pm}$are given by (6.20)-(6.22), and the coefficients $a^{ \pm}, b^{ \pm}$are given by $(6.25)-(6.28)$.

7. The second-order jump conditions. The first-order solution depends on one unknown function $\psi^{(1)}(k, \tau)$, the Fourier transform of the first-order displacement of the tangential discontinuity. The solution of the second-order interior equations depends, in addition, on two unknown functions $a_{0}^{ \pm}(k, \tau), b_{0}^{\mp}(k, \tau)$. In this section we study the second-order jump conditions. We show that they reduce to a singular linear system of algebraic equations for $\left(a_{0}^{ \pm}, b_{0}^{\mp}, \psi^{(2)}\right)$, where $\psi^{(2)}(k, \tau)$ is the Fourier transform of the second-order displacement of the tangential discontinuity. The imposition of solvability conditions on this system yields the equation for $\psi^{(1)}$ that we seek.

The second-order jump condition (4.4) may be written as

$$
\llbracket B \mathbf{u}^{(2)} \rrbracket-\varphi_{\theta}^{(2)} \llbracket f\left(\mathbf{u}^{(0)}\right)-\lambda h\left(\mathbf{u}^{(0)}\right) \rrbracket=\mathbf{q}
$$

where $\mathbf{q}$ is given by

$$
\mathbf{q}=\varphi_{\tau}^{(1)} \llbracket h\left(\mathbf{u}^{(0)}\right) \rrbracket+\varphi_{\theta}^{(1)} \llbracket(A-\lambda C) \mathbf{u}^{(1)} \rrbracket-\frac{1}{2} \llbracket \nabla^{2} g \cdot\left(\mathbf{u}^{(1)}, \mathbf{u}^{(1)}\right) \rrbracket
$$

Fourier transforming (7.1) with respect to $\theta$, we obtain

$$
\llbracket B \mathbf{U}^{(2)} \rrbracket-i k \psi^{(2)} \llbracket f\left(\mathbf{u}^{(0)}\right)-\lambda h\left(\mathbf{u}^{(0)}\right) \rrbracket=\mathbf{Q}
$$

where

$$
\mathbf{Q}(k, \eta, \tau)=\frac{1}{2 \pi} \int_{-\infty}^{+\infty} \mathbf{q}(\theta, \eta, \tau) e^{-i k \theta} d \theta
$$

In order to write out equation (7.2) explicitly, we evaluate at $\eta=0$ the expressions in (6.20)-(6.22) and (6.25)-(6.28) for the coefficients appearing in the solution (6.10) and 
(6.14) for $\mathbf{U}^{(2)}(k, \eta, \tau)$. This gives

$$
\begin{aligned}
& s_{1}^{ \pm}= \pm i \sigma_{1}^{ \pm} \frac{|k|}{k} \psi_{\tau}^{(1)}-\frac{c^{ \pm}}{k} \int_{-\infty}^{+\infty} \Omega_{1}(k, l) \psi^{(1)}(k-l) \psi^{(1)}(l) d l, \\
& s_{2}^{ \pm}=\sigma_{2}^{ \pm} \psi_{\tau}^{(1)} \text {, } \\
& s_{3}^{ \pm}=\mp i \sigma_{3}^{ \pm} \frac{|k|}{k} \psi_{\tau}^{(1)}+\frac{F_{0}^{ \pm}}{k} \int_{-\infty}^{+\infty} \Omega_{1}(k, l) \psi^{(1)}(k-l) \psi^{(1)}(l) d l, \\
& a^{-}= \begin{cases}a_{0}^{-} & \text {if } k<0 \\
i \alpha^{-} \psi_{\tau}^{(1)}+k \int_{-\infty}^{0} l \psi^{(1)}(k-l) \psi^{(1)}(l) d l & \text { if } k>0\end{cases} \\
& a^{+}= \begin{cases}i \alpha^{+} \psi_{\tau}^{(1)}+k \int_{0}^{+\infty} l \psi^{(1)}(k-l) \psi^{(1)}(l) d l & \text { if } k<0 \\
a_{0}^{+} & \text {if } k>0\end{cases} \\
& b^{-}= \begin{cases}-i \alpha^{-} \psi_{\tau}^{(1)}+k \int_{0}^{+\infty} l \psi^{(1)}(k-l) \psi^{(1)}(l) d l & \text { if } k<0 \\
b_{0}^{-} & \text {if } k>0\end{cases} \\
& b^{+}= \begin{cases}b_{0}^{+} & \text {if } k<0 \\
-i \alpha^{+} \psi_{\tau}^{(1)}+k \int_{-\infty}^{0} l \psi^{(1)}(k-l) \psi^{(1)}(l) d l & \text { if } k>0\end{cases}
\end{aligned}
$$

where

$$
\begin{aligned}
\sigma_{1}^{ \pm} & =\frac{\left(c^{ \pm}\right)^{2}+\left(F_{0}^{ \pm}\right)^{2}}{\left(c^{ \pm}\right)^{2}-\left(F_{0}^{ \pm}\right)^{2}}, \\
\sigma_{2}^{ \pm} & =\frac{\left(F_{0}^{ \pm}\right)^{2}\left(c^{ \pm}-u_{0}^{ \pm}\right)}{\lambda\left(\left(c^{ \pm}\right)^{2}-\left(F_{0}^{ \pm}\right)^{2}\right)}, \\
\sigma_{3}^{ \pm} & =\frac{2 c^{ \pm} F_{0}^{ \pm}}{\left(c^{ \pm}\right)^{2}-\left(F_{0}^{ \pm}\right)^{2}}, \\
\Omega_{1}(k, l) & =((k-l) l-|k-l||l|) l .
\end{aligned}
$$

Using (6.10) and (6.14) in (7.2), and simplifying the result with the help of these equations, we obtain two algebraic systems: the first one is for $a_{0}^{+}, b_{0}^{-}$, and $k \psi^{(2)}$ when $k>0$; and the second one is for $a_{0}^{-}, b_{0}^{+}$, and $k \psi^{(2)}$ when $k<0$.

First, we consider the case $k>0$. Then, equation (7.2) gives

$$
\left(B^{+} \mathbf{R}^{+},-B^{-} \overline{\mathbf{R}}^{-},-i \llbracket f\left(\mathbf{u}^{(0)}\right)-\lambda h\left(\mathbf{u}^{(0)}\right) \rrbracket\right)\left(\begin{array}{c}
a_{0}^{+} \\
b_{0}^{-} \\
k \psi^{(2)}
\end{array}\right)=\mathbf{Q}^{\prime}
$$

where

$$
\mathbf{Q}^{\prime}=\mathbf{Q}-[B S]+a^{-} B^{-} \mathbf{R}^{-}-b^{+} B^{+} \overline{\mathbf{R}}^{+}
$$


Writing out this system explicitly, and using (5.13) to simplify the result, we obtain that

$$
\begin{aligned}
& \left(\begin{array}{ccc}
i\left(c^{+} u_{0}^{+}+\left(F_{0}^{+}\right)^{2}\right) & i\left(c^{-} u_{0}^{-}+\left(F_{0}^{-}\right)^{2}\right) & -i \llbracket u_{0}^{2}-F_{0}^{2}-\lambda u_{0} \rrbracket \\
\left(c^{+}\right)^{2}-\left(F_{0}^{+}\right)^{2} & -\left(c^{-}\right)^{2}+\left(F_{0}^{-}\right)^{2} & 0 \\
i \lambda F_{0}^{+} & i \lambda F_{0}^{-} & i \lambda\left[F_{0}\right] \\
0 & 0 & 0 \\
i c^{+} & i c^{-} & -i\left[u_{0}\right]
\end{array}\right)\left(\begin{array}{c}
a_{0}^{+} \\
b_{0}^{-} \\
k \psi^{(2)}
\end{array}\right) \\
& =\left(\begin{array}{c}
\llbracket \frac{\left(F_{0}^{2}+c u\right)\left(3 \lambda c-2 F_{0}^{2}\right)}{2 \lambda\left(c^{2}-F_{0}^{2}\right)} \rrbracket \psi_{\tau}^{(1)} \\
-i \lambda\left(c^{+}+c^{-}\right) \int_{-\infty}^{+\infty} \Lambda_{1} \psi^{(1)}(k-l) \psi^{(1)}(l) d l \\
\frac{i}{2 \lambda}\left(\lambda\left(c^{+}+c^{-}\right)+2\left(F_{0}^{+}+F_{0}^{-}\right)\right) \psi_{\tau}^{(1)} \\
-\llbracket c^{2}-F_{0}^{2} \rrbracket k \int_{-\infty}^{0} l \psi^{(1)}(k-l) \psi^{(1)}(l) d l \\
\llbracket \frac{F_{0}\left(3 \lambda c-2 F_{0}^{2}\right)}{2\left(c^{2}-F_{0}^{2}\right)} \rrbracket \psi_{\tau}^{(1)} \\
-i \lambda\left(F_{0}^{+}+F_{0}^{-}\right) \int_{-\infty}^{+\infty} \Lambda_{1} \psi^{(1)}(k-l) \psi^{(1)}(l) d l \\
\llbracket \frac{\lambda c^{2}+2 u_{0} F_{0}^{2}}{2 \lambda\left(c^{2}-F_{0}^{2}\right)} \rrbracket \psi_{\tau}^{(1)} \\
-i\left(c^{+}+c^{-}\right) \int_{-\infty}^{+\infty} \Lambda_{1} \psi^{(1)}(k-l) \psi^{(1)}(l) d l
\end{array}\right),
\end{aligned}
$$

where

$$
\Lambda_{1}=\frac{1}{2}(3 k|l|-k l-2 l|l|) .
$$

The linear system (7.4) is solvable if and only if the rank of its augmented matrix is less than or equal to 3 . To determine this rank, we simplify the equations. We left-multiply the system by the vector

$$
\frac{i}{c^{+} c^{-}-F_{0}^{+} F_{0}^{-}}\left(-1,0, \frac{F_{0}^{+}+F_{0}^{-}}{\lambda}, 0, u_{0}^{+}+u_{0}^{-}-\lambda\right)^{T} .
$$

This gives the equation

$$
\begin{aligned}
a_{0}^{+}+b_{0}^{-}= & -i \frac{3 \lambda\left(c^{+}+c^{-}\right)-2\left(\left(F_{0}^{+}\right)^{2}+\left(F_{0}^{-}\right)^{2}\right)}{\lambda\left[c^{2}-F_{0}^{2}\right]} \psi_{\tau}^{(1)} \\
& -2 \int_{-\infty}^{+\infty} \Lambda_{1} \psi^{(1)}(k-l) \psi^{(1)}(l) d l .
\end{aligned}
$$

On the other hand, using (5.13) in the second equation of the system (7.4), we find that

$$
\begin{aligned}
a_{0}^{+}+b_{0}^{-}= & i \frac{\lambda\left(c^{+}+c^{-}\right)+2\left(\left(F_{0}^{+}\right)^{2}+\left(F_{0}^{-}\right)^{2}\right)}{\lambda\left[c^{2}-F_{0}^{2}\right]} \psi_{\tau}^{(1)} \\
& -2 k \int_{-\infty}^{0} l \psi^{(1)}(k-l) \psi^{(1)}(l) d l .
\end{aligned}
$$

Combining (7.5) and (7.6), we get the solvability condition

$$
i \llbracket \frac{c}{c^{2}-F_{0}^{2}} \rrbracket \psi_{\tau}^{(1)}+\int_{-\infty}^{+\infty}(2 k|l|-k l-l|l|) \psi^{(1)}(k-l) \psi^{(1)}(l) d l=0 .
$$


Then, solving (7.5) or (7.6) for $b_{0}^{-}$and substituting the result into the remaining two equations of the system, we find that both equations are equivalent to the equation

$$
a_{0}^{+}+k \psi^{(2)}=-i \frac{3 \lambda c^{+}-2\left(F_{0}^{+}\right)^{2}}{\lambda\left[c^{2}-F_{0}^{2}\right]} \psi_{\tau}^{(1)}-\int_{-\infty}^{+\infty} \Lambda_{1} \psi^{(1)}(k-l) \psi^{(1)}(l) d l .
$$

Thus, when $k>0$, the system (7.4) is solvable if and only if $\psi^{(1)}(k, \tau)$ satisfies Eq. (7.7), and then the rank of the augmented matrix of the system is equal to 2 .

The case $k<0$ is similar. The jump condition (7.2) gives the algebraic system

$$
\left(B^{+} \overline{\mathbf{R}}^{+},-B^{-} \mathbf{R}^{-},-i \llbracket f\left(\mathbf{u}^{(0)}\right)-\lambda h\left(\mathbf{u}^{(0)}\right) \rrbracket\right)\left(\begin{array}{c}
b_{0}^{+} \\
a_{0}^{-} \\
k \psi^{(2)}
\end{array}\right)=\mathbf{Q}^{\prime \prime},
$$

where

$$
\mathbf{Q}^{\prime \prime}=\mathbf{Q}-[B S]+b^{-} B^{-} \overline{\mathbf{R}}^{-}-a^{+} B^{+} \mathbf{R}^{+} .
$$

Proceeding as before, we find that, when (5.13) holds, this system (7.2) is solvable if and only if

$$
i \llbracket \frac{c}{c^{2}-F_{0}^{2}} \rrbracket \psi_{\tau}^{(1)}+\int_{-\infty}^{+\infty}(2 k|l|+k l-l|l|) \psi^{(1)}(k-l) \psi^{(1)}(l) d l=0 .
$$

In that case, the system is equivalent to the two equations

$$
\begin{aligned}
b_{0}^{+}+a_{0}^{-}= & -i \frac{\lambda\left(c^{+}+c^{-}\right)+2\left(\left(F_{0}^{+}\right)^{2}+\left(F_{0}^{-}\right)^{2}\right)}{\lambda\left[c^{2}-F_{0}^{2}\right]} \psi_{\tau}^{(1)} \\
& -2 k \int_{0}^{+\infty} l \psi^{(1)}(k-l) \psi^{(1)}(l) d l, \\
b_{0}^{+}+k \psi^{(2)}= & -i \frac{3 \lambda c^{+}-2\left(F_{0}^{+}\right)^{2}}{\lambda\left[c^{2}-F_{0}^{2}\right]} \psi_{\tau}^{(1)} \\
& -\int_{-\infty}^{+\infty} \Lambda_{2} \psi^{(1)}(k-l) \psi^{(1)}(l) d l,
\end{aligned}
$$

where

$$
\Lambda_{2}=\frac{1}{2}(3 k|l|+k l-2 l|l|) .
$$

Equations (7.7) for $k>0$ and (7.8) for $k<0$ are the solvability conditions for the second-order perturbation equations.

8. Summary. We have derived an asymptotic solution that describes the propagation of a weakly nonlinear, unidirectional surface wave on a tangential MHD discontinuity. The wave motion is governed by the incompressible MHD equations (3.1), and the states on either side of the discontinuity are given by (3.2) and (3.6). The linearized phase velocity $\lambda$ of the surface wave is a root of equation (5.14). The location of the tangential discontinuity is given by

$$
y=\varepsilon \varphi^{(1)}(x-\lambda t, \varepsilon t)+O\left(\varepsilon^{2}\right)
$$


as $\varepsilon \rightarrow 0$ with $t=O\left(\varepsilon^{-1}\right)$. The leading order field components may be expressed in terms of $\varphi^{(1)}$ using the results of the previous sections. The Fourier transform of the leading order perturbation $\varphi^{(1)}(\theta, \tau)$ in the location of the discontinuity,

$$
\psi^{(1)}(k, \tau)=\frac{1}{2 \pi} \int_{-\infty}^{+\infty} \varphi^{(1)}(\theta, \tau) e^{-i k \theta} d \theta
$$

satisfies (7.7) in $k>0$ and (7.8) in $k<0$. These equations may be written in a more compact form as

$$
\delta \psi_{\tau}^{(1)}(k, \tau)=i \operatorname{sgn} k \int_{-\infty}^{+\infty} \tilde{\Lambda}(k-l, l) \psi^{(1)}(k-l, \tau) \psi^{(1)}(l, \tau) d l,
$$

where

$$
\tilde{\Lambda}(k, l)=\operatorname{sgn}(k+l)\{2(k+l)|l|-|k+l| l-l|l|\} .
$$

The constant $\delta$ is given by

$$
\begin{aligned}
\delta & =\llbracket \frac{c}{c^{2}-F_{0}^{2}} \rrbracket \\
& =\frac{c^{+}}{\left(c^{+}\right)^{2}-\left(F_{0}^{+}\right)^{2}}-\frac{c^{-}}{\left(c^{-}\right)^{2}-\left(F_{0}^{-}\right)^{2}},
\end{aligned}
$$

where $c^{ \pm}$is defined in (5.9). We may equivalently replace $\tilde{\Lambda}$ in (8.1) by the symmetrized kernel

$$
\Lambda(k, l)=\frac{1}{2}[\tilde{\Lambda}(k, l)+\tilde{\Lambda}(l, k)] .
$$

This symmetrized kernel is identical to the one in (1.5), as may be checked by a consideration of the different possible choices for the signs of $k, l$, and $k+l$. Equations (8.1)-(8.2) are therefore equivalent to (1.4)-(1.5) after an appropriate rescaling.

\section{REFERENCES}

[1] G. Alì, J. K. Hunter, and D. F. Parker, Hamiltonian equations for scale-invariant waves, Stud. Appl. Math., 108 (2002), 305-321.

[2] M. Artola, and A. J. Majda, Nonlinear development of instabilities in supersonic vortex sheets I. The basic kink modes, Physica D, 28 (1987), 253-281.

[3] M. Artola, and A. J. Majda, Nonlinear development of instabilities in supersonic vortex sheets. II. Resonant interaction among kink modes, SIAM J. Appl. Math., 49 (1989), 1310-1349.

[4] S. Benzoni-Gavage, Stability of subsonic planar phase boundaries in a van der Waals fluid, Arch. Ration. Mech. Anal. 150 (1999), 23-55.

[5] M. F. Hamilton, Yu. A. Il'insky, and E. A. Zabolotskaya, Local and nonlocal nonlinearity in Rayleigh waves, J. Acoust. Soc. Am., 97 (1995), 882-890.

[6] M. F. Hamilton, Yu. A. Il'insky, and E. A. Zabolotskaya, Evolution equations for nonlinear Rayleigh waves, J. Acoust. Soc. Am., 97 (1995), 891-897.

[7] M. F. Hamilton, Yu. A. Il'insky, and E. A. Zabolotskaya, Nonlinear surface acoustic waves in crystals, J. Acoust. Soc. Am., 1052 (1999), 639-651.

[8] R. L. Higdon, Initial-boundary value problems for linear hyperbolic systems, SIAM Rev., 28 (1986), 177-217.

[9] J. K. Hunter, Nonlinear surface waves, Contemporary Mathematics, 100 (1989), 185-202.

[10] N. Kalyanasundaram, Nonlinear surface acoustic waves on an isotropic solid, Int. J. Engng. Sci., 19 (1981), 279-286.

[11] N. Kalyanasundaram, R. Ravindran and P. Prasad, Coupled amplitude theory of nonlinear surface acoustic waves, J. Acoust. Soc. Am., 72 (1982), 488-493. 
[12] H.-O. Kreiss, Initial boundary value problems for hyperbolic systems, Comm. Pure Appl. Math., 23 (1970), 277-298.

[13] L. D. Landau, and E. M. Lifschitz, Electrodynamics of Continuous Media, 2nd ed., Pergamon Press, New York, 1984.

[14] R. W. Lardner, Nonlinear surface waves on an elastic solid, Int. J. Engng. Sci., 21 (1983), 13311342 .

[15] R. W. Lardner, Nonlinear surface acoustic waves on an elastic solid of general anisotropy, J. Elast., 16 (1986), 63-73.

[16] A. J. Majda, The stability of multidimensional shock fronts. Mem. Amer. Math. Soc., 41 (1983), no. 275.

[17] A. J. Majda, The existence of multi-dimensional shock fronts, Mem. Amer. Math. Soc., 43 (1983), no. 281. AMS, Providence, 1983.

[18] A. J. Majda, Compressible Fluid Flow and Systems of Conservation Laws in Several Space Variables, Applied Mathematical Sciences, Vol. 53, Springer-Verlag, New York, 1984.

[19] A. J. Majda, and R. R. Rosales, A theory for spontaneous Mach stem formation in reacting shock fronts. I. The basic perturbation analysis, SIAM J. Appl. Math., 43 (1983), 1310-1334.

[20] A. J. Majda, and R. R. Rosales, A theory for spontaneous Mach stem formation in reacting shock fronts. II. Steady wave bifurcation and the evidence for breakdown, Stud. Appl. Math., 71 (1984), $117-148$.

[21] P. J. Olver, Hamiltonian and non-Hamiltonian models for water waves, Trends and Applications of Pure Mathematics to Mechanics (Palaiseau, 1983), 273-290, Lecture Notes in Phys., 195, SpringerVerlag, Berlin, 1984.

[22] D. F. Parker, Waveform evolution for nonlinear surface acoustic waves, Int. J. Engng. Sci., 26 (1988), 59-75.

[23] D. F. Parker, and F. M. Talbot, Analysis and computation for nonlinear elastic surface waves of permanent form, J. Elast., 15 (1985), 389-426.

[24] R. R. Rosales, Stability theory for shocks in reacting media: Mach stems in detonation waves, Lectures in Applied Mathematics 24 (1986), 431-465.

[25] R. Sakamoto, Mixed problems for hyperbolic equations. I. Energy inequalities, J. Math. Kyoto Univ., 10 (1970), 349-373.

[26] R. Sakamoto, Mixed problems for hyperbolic equations. II. Existence theorems with zero initial datas and energy inequalities with initial datas, J. Math. Kyoto Univ., 10 (1970), 403-417.

[27] D. Serre, Systems of Conservation Laws. 2. Geometric Structures, Oscillations, and InitialBoundary Value Problems, Cambridge University Press, Cambridge, 2000.

[28] E. Tadmor, Convergence of spectral methods for nonlinear conservation laws, SIAM J. Numer. Anal. 26 (1989), 30-44.

[29] E. A. Zabolotskaya, Nonlinear propagation of plane and circular Rayleigh waves in isotropic solids, J. Acoust. Soc. Am. 91 (1992), 2569-2575. 